\title{
Endovascular Aortic Repair under the Monitored Anesthesia Care with Dexmedetomidine without Local Anesthesia: A Retrospective Study
}

\author{
Ki Tae Jung, M.D. ${ }^{1,2}$, Sang Hun Kim, M.D., Ph.D. ${ }^{1,2}$, Hong Ju Seo, M.D., Ph.D. ${ }^{3}$, \\ Jeong Hwan Chang, M.D., Ph.D. ${ }^{4}$ \\ ${ }^{1}$ Department of Anesthesiology and Pain Medicine, \\ Chosun University, School of medicine, Republic of Korea \\ ${ }^{2}$ Department of Anesthesiology and Pain Medicine, \\ Chosun University Hospital, Republic of Korea \\ ${ }^{3}$ Department of Thoracic and Cardiovascular Surgery, \\ Chosun University, School of Medicine, Republic of Korea \\ ${ }^{4}$ Department of Surgery, Chosun University, \\ School of Medicine, Republic of Korea
}

\begin{abstract}
:
Background: Dexmedetomidine has cardiac protective effect and unique sedative as well as analgesic properties while having minimal respiratory effects, making it suitable for monitored anesthesia care (MAC). We report 13 experiences of performing Endovascular aortic repair (EVAR) under MAC with dexmedetomidine.

Methods: We retrospectively reviewed records of 24 patients who underwent EVAR from August 1, 2011, until April 30, 2014. 13 patients, who underwent MAC using dexmedetomidine without local anesthesia, were enrolled.

Results: MAC with dexmedetomidine, remifentanil and propofol was performed in 8 patients, MAC with dexmedetomidine and remifentanil was performed in 5 patients. All patients showed the intraoperative stable hemodynamics. Their peripheral oxygen saturation was maintained above $90 \%$ and bispectral index was maintained from 80 to 40. Transient hypoxemia, hypotension, and bradycardia were occurred at an early period of MAC in 4, 3, and 4 patients, respectively, which was completely resolved by intermittent bolus injection of naloxone, phenylephrine, ephedrine, or atropine. Patients were awakened and following simple commands within about 20 minute after the end of MAC.
\end{abstract}

Conclusions: This retrospective analysis demonstrates that MAC with combination technique of dexmedetomidine and remifentanil is safe and effective to provide the intraoperative stable hemodynamics and respiratory condition during the EVAR.

Key words: Dexmedetomidine, Endovascular Aortic Repair, Monitored Anesthesia Care, Remifentanil.

\section{INTRODUCTION}

The surgical treatment of aortic aneurysms or dissection has traditionally involved a conventional open surgical repair, which is associated with relatively high mortality and morbidity. ${ }^{1}$ With the successful development of the minimally invasive endovascular technique, which is much less invasive and significantly less strain on the heart and vital organs, endovascular aortic repair (EVAR) is gaining widespread acceptance and can be offered to patients tolerable outcomes compared with open repair. ${ }^{2}$

During EVAR, various anesthetic techniques such as general anesthesia and locoregional anesthesia with or without sedation have been used.$^{3-6}$ However, the ideal anesthetic and monitoring techniques have not yet been scientifically evaluated and determined as extensively as in vascular surgical procedures. ${ }^{1,7}$ Many surgeons seem to decide either locoregional anesthesia or general anesthesia depending on the status of each institution. Previous reports showed that the local anesthesia is more 
feasible for EVAR than general anesthesia because of minimal surgical incision and less complications. ${ }^{3-6}$ Although local anesthesia can reduced the painful stimuli during procedure of EVAR, an additional analgesic and sedative is needed to ensure an acceptable level of patient comfort and cardiorespiratory stability. The sole or combination regimens of propofol, remifentanil, ketamine, or midazolam have been commonly used in the past. ${ }^{8,9}$ However, these agents can induce variability of patient response and respiratory complications such as dose-dependent respiratory depression, hypoxemia, and apnea, which create the need for a sedative drug which can be used safely in both healthy and high-risk patients, with limited adverse effects. ${ }^{10}$ Candiotti et al. ${ }^{10}$ suggested that dexmedetomidine, selective $\alpha 2$-adrenergic receptor agonist, is an effective sedative for monitored anesthesia care (MAC) by reducing the opioid requirements and the incidence of respiratory depression as well as providing the better patient satisfaction than placebo rescued with midazolam and fentanyl. Brown et al. ${ }^{11}$ investigated the effect of dexmedetomidine under epidural anesthesia compared with general anesthesia alone for EVAR, and they suggested that the dexmedetomidine under epidural anesthesia would be useful for procedure of EVAR.

However, there are no literatures that investigated the effectiveness of dexmedetomidine without local infiltration for the procedure of EVAR. Therefore, the authors report the clinical experiences of MAC with dexmedetomidine in patients underwent EVAR without local anesthesia, and reviewed the articles using dexmedetomidine for MAC in fields of endovascular procedures.

\section{Material AND Methods}

After obtaining approval for this study from the Institutional Review Board, a retrospective chart review and analysis were conducted on patients who underwent endovascular aortic repair (EVAR) without local anesthesia under monitored anesthesia care (MAC) with dexmedetomidine, from August 1, 2011, until April 30, 2014. We excluded the patients who were preoperatively deteriorate, did not received dexmedetomidine, or underwent EVAR under the general and regional anesthesia.

We analyzed systolic blood pressure (SBP), diastolic blood pressure (DBP), and heart rate (HR), respiratory rate (RR), bispectral index score (BIS) and peripheral oxygen saturation $\left(\mathrm{SpO}_{2}\right)$, which were recorded at each time point, as follows: anesthesia start as baseline value and every 5 minutes after starting anesthesia until the end of the procedures. In addition, we analyzed SBP, DBP, HR and RR at postoperative 1,2,3, and 4 hour. We also recorded and analyzed the patient's demographic data [age, sex, and American Society of Anesthesiologists (ASA) score], the incidence of perioperative adverse effects (hypertension, hypotension, bradycardia, tachycardia, bradypnea, apnea, and delirium), and the mean intraoperative infusion rate of dexmedetomidine, remifentanil, and propofol. And then, we allocated two groups with enrolled patients according to using propofol and analyzed the above mentioned parameters.

All measurements are presented as mean \pm standard deviation or number of patients. The data were analyzed using SPSS ver. 21.0 for Windows (SPSS, Inc., Chicago, IL, USA). First, all data were analyzed using simple descriptive statistics. Second, to compare two groups, The Mann-Whitney U test was used to compare means and the Fisher exact test was used to compare categorical variables. Statistical significance was designated at $\mathrm{p}<0.05$.

\section{RESUlTS}

EVAR was performed in 24 patients during this period, 11 patients were excluded because of general and regional anesthesia, and the preoperative deteriorated conditions. Finally, 13 patients were enrolled in this study: the MAC with dexmedetomidine, remifentanil, and propofol (group PRD) was performed in 8 patients while the MAC with dexmedetomidine and remifentanil (group RD) was performed in 5 patients. A summary of the clinical characteristics of the enrolled patients is shown in Table I. There were no significant differences between groups.

Table1. Baseline clinical characteristics in two groups

\begin{tabular}{|l|c|c|c|}
\hline & Both $(\mathbf{n}=\mathbf{1 3})$ & Group PRD $(\mathbf{n}=\mathbf{8})$ & Group RD $(\mathbf{n}=\mathbf{5})$ \\
\hline Gender $(\mathrm{male} / \mathrm{female} ; \mathrm{n})$ & $13 / 0$ & $8 / 0$ & $5 / 0$ \\
\hline Age $($ Year) & $68.5 \pm 6.8$ & $69.0 \pm 3.0$ & $67.80 \pm 1.5$ \\
\hline Height $(\mathrm{cm})$ & $167.5 \pm 7.0$ & $167.3 \pm 3.2$ & $167.8 \pm 0.6$ \\
\hline Weight $(\mathrm{Kg})$ & $73.2 \pm 9.8$ & $76.6 \pm 3.3$ & $67.6 \pm 3.9$ \\
\hline Diagnosis (AAA/AAD/TAAA/TAAD; $\mathrm{n})$ & $10 / 1 / 1 / 1$ & $5 / 1 / 1 / 1$ & $5 / 0 / 0 / 0$ \\
\hline
\end{tabular}


Values are presented as mean \pm standard deviation (SD) or number of patients. Group PRD is the patients who were controlled with propofol, remifentanil, and dexmedetomidine. Group RD is the patients who were controlled with remifentanil, and dexmedetomidine. AAA, abdominal aortic aneurysm; AAD, abdominal aortic dissection; TAAA, thoracic abdominal aortic aneurysm; TAAD, thoracic abdominal aortic dissection

All patients showed the stable hemodynamics, which were maintained within normal ranges, in perioperative period (Figure. 1). There were no significant differences of intraoperative SAP and DAP between two groups, but there were the significantly more decreased DAP at postoperative 1 and 2 hours in group RD ( $p<0.05$, Figure. $1, A)$. The HR in group RD was significantly more decreased than in group PRD in perioperative period ( $p<0.05$, Figure. $1, B)$. The overall mean RR was maintained more than 10 breaths per minute (BPM) although the RR in group RD was significantly slower than in group PRD at 90,120, and 150 minute after anesthetic induction and at postoperative 4 hour $(\mathrm{p}<0.05$, Figure. 1, C). . Their peripheral oxygen saturation was maintained above $90 \%$ and bispectral index (BIS) was maintained from 80 to 40 during procedure (Figure. 1, D). The BIS in group RD was significantly higher than in group PRD at 10,30, 60, 90, and 120 minute after anesthetic induction $(\mathrm{p}<0.05$, Figure 1, D). Adequate sedation for the endovascular procedure was achieved in all cases.
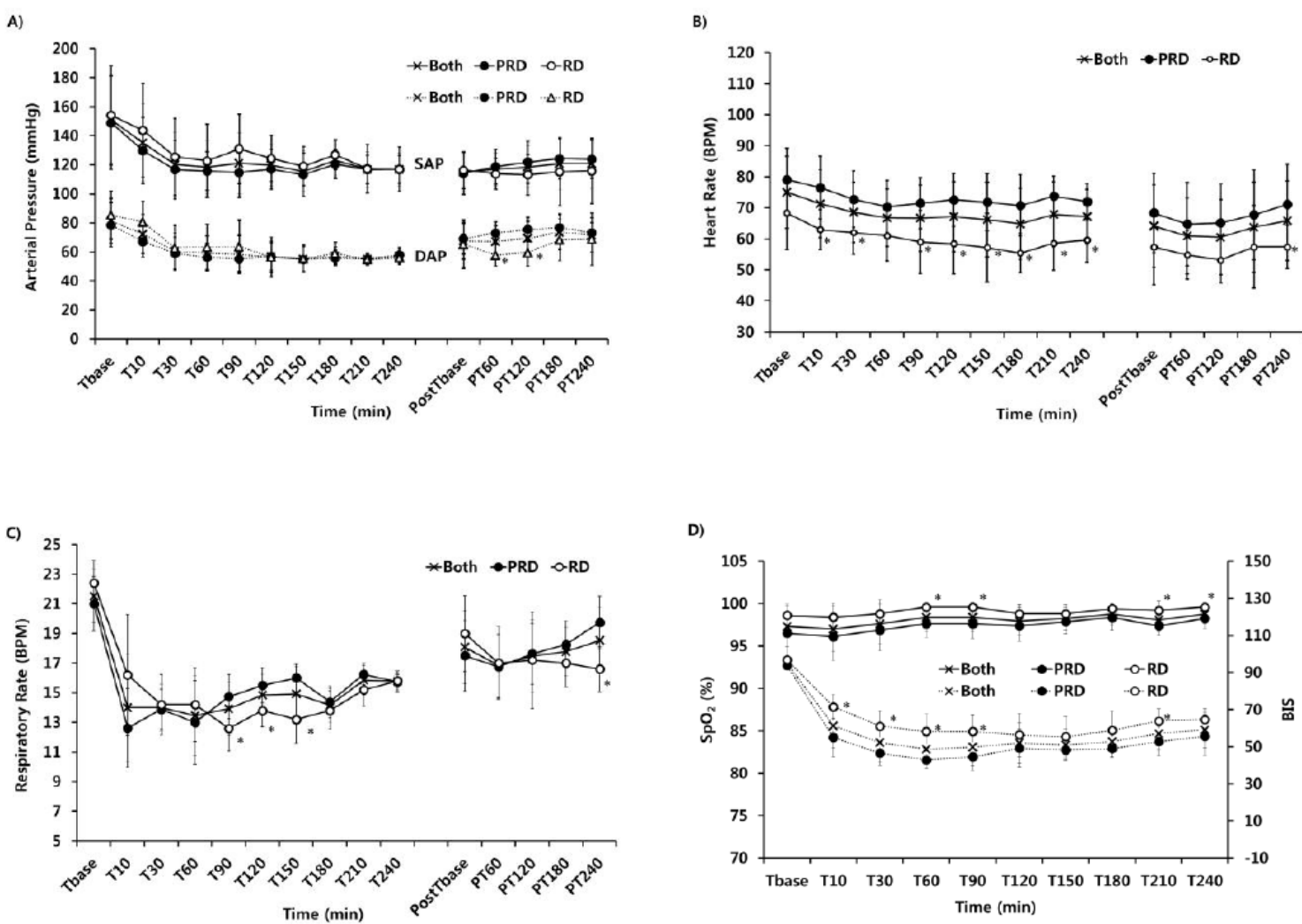

Figure1. The perioperative changes of the arterial pressure $(A)$, heart rate $(B)$, respiratory rate $(C)$, and peripheral oxygen saturation $\left(\mathrm{SpO}_{2}\right)$ and bispectral index score $(B I S)(D)$. Group PRD is the patients who were controlled with propofol, remifentanil, and dexmedetomidine. Group RD is the patients who were controlled with remifentanil and dexmedetomidine. SAP, systolic arterial pressure; DAP, diastolic arterial pressure; Tbase, before starting anesthesia; T10, 10 minute after induction; T30 T240, time per 30 minute after anesthesia; PostTbase, end of anesthesia; PT $60 \sim$ PT240, time per 60 minute after end of anesthesia. ${ }^{*} P<0.05$, statistically significance compared with group PRD.

Group RD showed the significantly larger infusion rate of remifentanil and lesser infusion rate of dexmedetomidine ( $\mathrm{P}<0.05$, Table II). There were no significant differences in mean operation time, fluid intake, and estimated blood loss between two groups except of urine output, which was significantly larger in group PRD than in group RD ( $\mathrm{p}<0.05$, Table III). Patients were awakened and following simple commands within mean about 20 minute after the end of MAC (Table III). There was transient adverse effects such as bradypnea, hypotension, and bradycardia were occurred at an early period of MAC in 4, 3, and 4 patients, respectively, which was completely resolved by 
intermittent bolus injection of naloxone, phenylephrine, ephedrine, or atropine (Table III). There was no significant difference between groups, but bradypnea was only occurred in group PRD. No patient required conversion to general anesthesia or an open repair.

Table2. Intraoperative mean dosages of dexmedetomidine, remifentanil, and propofol

\begin{tabular}{|l|c|c|c|}
\hline & Both $(\mathbf{n}=\mathbf{1 3})$ & Group PRD $(\mathbf{n}=8)$ & Group RD (n = 5) \\
\hline dexmedetomidine $(\mu \mathrm{g} / \mathrm{kg} / \mathrm{hr})$ & $1.6 \pm 1.5$ & $2.0 \pm 1.4^{*}$ & $1.1 \pm 1.4$ \\
\hline Remifentanil $(\mathrm{ng} / \mathrm{ml})$ & $2.1 \pm 0.5$ & $2.0 \pm 0.4$ & $2.2 \pm 0.5^{*}$ \\
\hline Propofol $(\mu \mathrm{g} / \mathrm{ml})$ & $0.5 \pm 0.6$ & $1.0 \pm 0.5^{*}$ & $0.0 \pm 0.0$ \\
\hline
\end{tabular}

Values are presented as mean \pm standard deviation $(S D)$. Group PRD is the patients who were controlled with propofol, remifentanil, and dexmedetomidine. Group RD is the patients who were controlled with remifentanil, and dexmedetomidine. ${ }^{*} P<0.05$, statistically significance compared with group PRD.

Table3. Comparison of intraoperative data between two groups

\begin{tabular}{|l|c|c|c|}
\hline & Both (n= 13) & Group PRD (n = 8) & Group RD (n = 5) \\
\hline Operation time (min) & $204.6 \pm 59.6$ & $204.4 \pm 69.3$ & $205.0 \pm 47.6$ \\
\hline Fluid requirement (ml) & $2003.8 \pm 593.9$ & $2087.5 \pm 749.2$ & $1870.0 \pm 198.8$ \\
\hline Estimated blood loss (ml) & $316.2 \pm 102.4$ & $288.8 \pm 91.1$ & $360.0 \pm 114.0$ \\
\hline Urine output (ml) & $1223.1 \pm 775.8$ & $1562.5 \pm 810.5^{*}$ & $680.0 \pm 238.7$ \\
\hline Minimum SpO (\%) & $96.5 \pm 2.6$ & $95.4 \pm 2.6$ & $98.4 \pm 1.3$ \\
\hline Wake up time (min) & $20.6 \pm 7.6$ & $23.5 \pm 8.0$ & $16.0 \pm 4.2$ \\
\hline Morbidity & & & $2(40)$ \\
\hline Hypotension (n) & $3(23.1)$ & $1(12.5)$ & $2(40)$ \\
\hline Bradycardia (n) & $4(30.8)$ & $2(25)$ & 0 \\
\hline Transient bradypnea (n) & $4(30.8)$ & $4(50)$ & 0 \\
\hline Postoperative delirium (n) & $1(7.7)$ & $1(12.5)$ & \\
\hline
\end{tabular}

Values are presented as mean \pm standard deviation (SD) or number of patients (\%). Group PRD is the patients who were controlled with propofol, remifentanil, and dexmedetomidine. Group RD is the patients who were controlled with remifentanil, and dexmedetomidine. $\mathrm{SpO}_{2}$, peripheral oxygen saturation. ${ }^{*} \mathrm{P}<0.05$, statistically significance compared with group PRD.

\section{DisCUSSION}

In this retrospective study, the data showed that the dexmedetomidine was infused a loading dose of 1 $\mu \mathrm{g} / \mathrm{kg}$ over 10 minutes, followed by a continuous dose $0.5-4.0 \mu \mathrm{g} / \mathrm{kg} / \mathrm{h}$, and remifentanil was continuously infused before starting operation to prevent unexpected movements and to ensure patient comfort instead of local anesthetics infiltration into the groin. Our patients maintained spontaneous respiration throughout the procedures under MAC, and the general surgeon could implant the stent in the proper position without significant hemodynamic and pulmonary instability. Most patients' respiratory rate was maintained above $10 \mathrm{BPM}$, and $\mathrm{SpO}_{2}$ was $92-100 \%$.

Over the last decade, there has been a paradigm shift in the treatment of aortic aneurysm from open repair to endovascular aortic repair (EVAR), which was first described in 1991. Many kinds of anesthetic techniques, such as general anesthesia, regional anesthesia, and local anesthetic infiltration with/without sedation, have been used for EVAR. However, the anesthetic management of patients with an aortic aneurysm remains a challenge to the anesthesiologists because there is lacking on the strong evidence of mortality or morbidity benefit of one technique over another. Generally, most anesthesiologists and surgeons decided the anesthetic method according to patient co-morbidities, duration and method of surgery, use of antiplatelet or anticoagulant drugs, the ability to lay flat and patient preference.

Even though most reports have shown that the local infiltration or regional anesthesia was associated with a lower incidence of perioperative complications compared with general anesthesia, ${ }^{5,12-14}$ the patients underwent local infiltration or regional anesthesia may require additional sedation or analgesia because of agitation secondary to restlessness and pain from lying in one position for a prolonged period of time. ${ }^{5,15}$ Therefore, maintenance of proper drug level and adequate depth of analgesia and sedation without motion during the diagnostic or therapeutic procedures is essential and are the most important considering factor for safe endovascular procedure. For these purpose, the several sedative such as midazolam, propofol, ketamine, and dexmedetomidine has been used for MAC during endovascular, vascular, or diagnostic procedures. Varty et al. ${ }^{16}$ recommended that a benzodiazepine or propofol for anxiety, and fentanyl or remifentanil for pain is helpful with local 
anesthesia for elective procedure of EVAR, but anesthesiologists need to titrate this carefully with airway control because of a potential respiratory depression effect. Form this point of view, dexmedetomidine, a more specific and selective $\alpha 2$-adrenergic receptor agonist might be suitable because dexmedetomidine has several specific characteristics such as anxiolytic and sedative effect, analgesic effect, neurocardioprotection, and minimal effect on ventilation. ${ }^{17-25}$ Dexmedetomidine produces an unusually cooperative form of sedation, in which patients easily transit from sleep to wakefulness and cooperate with procedures when aroused, and then back to sleep when not stimulated. ${ }^{17}$ In cardiovascular effects, the initial response to the loading dose of dexmedetomidine with rapid injection may be a transient hypertension by vasoconstriction, and follow the mild to moderate bradycardia and hypotension, which were not usually needed by pharmacologic intervention. ${ }^{10,}{ }^{19,}{ }^{24-26}$ Our patients also showed the acceptable perioperative hemodynamic changes like the above mentioned reports, but the bradypnea, which was required the injection of naloxone for respiratory stimulation, was only developed in patients who received propofol as an adjuvant of dexmedetomidine for sedation even though its dose was very low.

Interestingly, dexmedetomidine has a sparing analgesic effect in contrast with other sedatives, for which the reducing opioid requirements by $30 \%$ to $50 \%$ offered support. ${ }^{10,11,22,23}$ However, the sedation using the dexmedetomidine as well as other sedatives alone cannot provide sufficient analgesia if local infiltration or analgesics is not provided during diagnostic and interventional procedures. ${ }^{27}$ Therefore, supplemental analgesic or local anesthesia is required, ${ }^{28-31}$ and intraoperative remifentanil infusion may be safer because it provided the more significantly stable analgesia compared with the combination of intermittent fentanyl and midazolam ${ }^{28}$. In review article of Shukry and Miller, ${ }^{29}$ they demonstrated that dexmedetomidine $(0.5-5 \mu \mathrm{g} / \mathrm{kg}$ of loading dose and $0.2-10$ $\mu \mathrm{g} / \mathrm{kg} / \mathrm{h}$ of infusion dose) has been commonly used as a sedative and hypnotic for patients undergoing procedures without tracheal intubation, which was administrated in conjunction with local anesthesia, opioids, or sedatives. Heo et al. ${ }^{31}$ suggested that the half maximal effective concentration (EC50) of remifentanil reached target concentration for preventing cystoscope insertion pain was $1.33 \mathrm{ng} / \mathrm{ml}$ (1.44-2.48 ng/ml) when combined with dexmedetomidine (a loading dose of $1.0 \mu \mathrm{g} / \mathrm{kg}$ dexmedetomidine over 10 minutes, followed by a maintenance dose of $0.6 \mu \mathrm{g} / \mathrm{kg} / \mathrm{hr}$ ). In our cases, intraoperative pain was controlled with $2.1 \pm 0.5 \mathrm{ng} / \mathrm{ml}$ of remifentanil (the effect-site concentration), and these doses was enough to prevent the procedure-induced pain without local infiltrations. One of the interesting findings in our reports was the increased urine output in group which dexmedetomidine was used higher dose. Several reports suggested that dexmedetomidine-induced sympatholysis might result in prevention of acute kidney injury by increased urinary output and preserved creatinine levels and higher creatinine clearance without the need of high doses of diuretics. ${ }^{32-34}$ So, if there is needed the protection of renal function during EVAR, it may be helpful.

\section{Conclusion}

The monitored anesthesia care with combination technique of dexmedetomidine and remifentanil is a safe and effective way of providing anesthesia for the endovascular repair of aortic aneurysm and dissection, producing consistently tolerable operating conditions.

\section{ACKNOWLEDGEMENT}

This study was supported by research funds from Chosun University Hospital 2015.

\section{REFERENCES}

[1] De Virgilio C, Romero L, Donayre C, Meek K, Lewis RJ, Lippmann M et al. Endovascular abdominal aortic aneurysm repair with general versus local anesthesia: a comparison of cardiopulmonary morbidity and mortality rates. J Vasc Surg 2002; 36: 988-91.

[2] Riddell JM, Black JH, Brewster DC, Dunn PF. Endovascular abdominal aortic aneurysm repair. Int Anesthesiol Clin 2005; 43: 79-91.

[3] Edwards MS, Andrews JS, Edwards AF, Ghanami RJ, Corriere MA, Goodney PP et al. Results of endovascular aortic aneurysm repair with general, regional, and local/monitored anesthesia care in the American College of Surgeons National Surgical Quality Improvement Program database. J Vasc Surg 2011; 54: 1273-82.

[4] Franz R, Hartman J, Wright M. Comparison of anesthesia technique on outcomes of 
endovascular repair of abdominal aortic aneurysms: a five-year review of monitored anesthesia care with local anesthesia vs. general or regional anesthesia. J Cardiovasc Surg 2011; 52: 567-77.

[5] Verhoeven EL, Cina CS, Tielliu IF, Zeebregts CJ, Prins TR, Eindhoven GB et al. Local anesthesia for endovascular abdominal aortic aneurysm repair. J Vasc Surg 2005; 42: 402-9.

[6] Henretta JP, Hodgson KJ, Mattos MA, Karch LA, Hurlbert SN, Sternbach Y et al. Feasibility of endovascular repair of abdominal aortic aneurysms with local anesthesia with intravenous sedation. J Vasc Surg 1999; 29: 793-8.

[7] Aadahl P, Lundbom J, Hatlinghus S, Myhre HO. Regional anesthesia for endovascular treatment of abdominal aortic aneurysms. Journal of endovascular surgery : the official journal of the International Society for Endovascular Surgery 1997; 4: 56-61.

[8] Ghisi D, Fanelli A, Tosi M, Nuzzi M, Fanelli G. Monitored anesthesia care. Minerva Anestesiol 2005; 71: 533-8.

[9] Savoia G, Loreto M, Gravino E, Canfora G, Frangiosa A, Cortesano P et al. Monitored anesthesia care and loco-regional anesthesia. Vascular surgery use. Minerva Anestesiol 2005; 71: 539-42.

[10] Candiotti KA, Bergese SD, Bokesch PM, Feldman MA, Wisemandle W, Bekker AY. Monitored anesthesia care with dexmedetomidine: a prospective, randomized, double-blind, multicenter trial. Anesth Analg 2010; 110: 47-56.

[11] Brown BJ, Zakhary S, Rogers L, Ellis-Stoll C, Gable D, Ramsay MA. Use of dexmedetomidine versus general anesthesia for endovascular repair of abdominal aortic aneurysms. Proceedings 2006; 19: 213-5.

[12] Ruppert V, Leurs LJ, Steckmeier B, Buth J, Umscheid T. Influence of anesthesia type on outcome after endovascular aortic aneurysm repair: an analysis based on EUROSTAR data. J Vasc Surg 2006; 44: 16-21.

[13] Karthikesalingam A, Thrumurthy SG, Young EL, Hinchliffe RJ, Holt PJ, Thompson MM. Locoregional anesthesia for endovascular aneurysm repair. J Vasc Surg 2012; 56: 510-9.

[14] Bakker EJ, van de Luijtgaarden KM, van Lier F, Valentijn TM, Hoeks SE, Klimek M et al. General Anaesthesia is Associated with Adverse Cardiac Outcome after Endovascular Aneurysm Repair. Eur J Vasc Endovasc Surg 2012; 44: 121-5.

[15] Baril DT, Kahn RA, Ellozy SH, Carroccio A, Marin ML. Endovascular Abdominal Aortic Aneurysm Repair: Emerging Developments and Anesthetic Considerations. J Cardiothorac Vasc Anesth 2007; 21: 730-42.

[16] Varty K. Comments regarding 'Local anaesthesia for endovascular repair of infra-renal aortic aneurysms'. Eur J Vasc Endovasc Surg 2011; 42: 474.

[17] Venn RM, Grounds RM. Comparison between dexmedetomidine and propofol for sedation in the intensive care unit: patient and clinician perceptions. Br J Anaesth 2001; 87: 684-90.

[18] Martin E, Ramsay G, Mantz J, Sum-Ping ST. The role of the alpha2-adrenoceptor agonist dexmedetomidine in postsurgical sedation in the intensive care unit. J Intensive Care Med 2003; 18: $29-41$.

[19] Lee S, Kim BH, Lim K, Stalker D, Wisemandle W, Shin SG et al. Pharmacokinetics and pharmacodynamics of intravenous dexmedetomidine in healthy Korean subjects. J Clin Pharm Ther 2012; 37: 698-703.

[20] Ramsay MA, Luterman DL. Dexmedetomidine as a total intravenous anesthetic agent. Anesthesiology 2004; 101: 787-90.

[21] Ma D, Hossain M, Rajakumaraswamy N, Arshad M, Sanders RD, Franks NP et al. Dexmedetomidine produces its neuroprotective effect via the alpha 2A-adrenoceptor subtype. Eur J Pharmacol 2004; 502: 87-97.

[22] Venn RM, Bradshaw CJ, Spencer R, Brealey D, Caudwell E, Naughton C et al. Preliminary UK experience of dexmedetomidine, a novel agent for postoperative sedation in the intensive care unit. Anaesthesia 1999; 54: 1136-42.

[23] Arain SR, Ruehlow RM, Uhrich TD, Ebert TJ. The efficacy of dexmedetomidine versus morphine for postoperative analgesia after major inpatient surgery. Anesthesia and analgesia 2004; 98: 153-8. 
[24] Talke P, Chen R, Thomas B, Aggarwall A, Gottlieb A, Thorborg P et al. The hemodynamic and adrenergic effects of perioperative dexmedetomidine infusion after vascular surgery. Anesth Analg 2000; 90: 834-9.

[25] Snapir A, Posti J, Kentala E, Koskenvuo J, Sundell J, Tuunanen H et al. Effects of low and high plasma concentrations of dexmedetomidine on myocardial perfusion and cardiac function in healthy male subjects. Anesthesiology 2006; 105: 902-10.

[26] Gupta P, Joshi S, Jethava D, Kumar A. Dexmedetomidine ameliorates monitored anaesthesia care. Indian J Anaesth 2014; 58: 154-9.

[27] Huncke TK, Adelman M, Jacobowitz G, Maldonado T, Bekker A. A prospective, randomized, placebo-controlled study evaluating the efficacy of dexmedetomidine for sedation during vascular procedures. Vasc Endovascular Surg 2010; 44: 257-61.

[28] Bonfreschi V, Giuliani E, Malagnino FC, Navi A, Coppi G, Silingardi R et al. Analgesia during abdominal aortic aneurysm endovascular repair: remifentanil vs. fentanyl-midazolam--a randomized controlled trial. European journal of anaesthesiology 2009; 26: 782-7.

[29] Shukry M, Miller JA. Update on dexmedetomidine: use in nonintubated patients requiring sedation for surgical procedures. Therapeutics and clinical risk management 2010; 6: 111-21.

[30] Mester R, Easley RB, Brady KM, Chilson K, Tobias JD. Monitored anesthesia care with a combination of ketamine and dexmedetomidine during cardiac catheterization. Am J Ther 2008; 15: 24-30.

[31] Heo B, Kim M, Lee H, Park S, Jeong S. Optimal effect-site concentration of remifentanil when combined with dexmedetomidine in patients undergoing cystoscopy. Korean J Anesthesiol 2014; 66: 39-43.

[32] Kulka PJ, Tryba M, Zenz M. Preoperative alpha2-adrenergic receptor agonists prevent the deterioration of renal function after cardiac surgery: results of a randomized, controlled trial. Crit Care Med 1996; 24: 947-52.

[33] Herr DL, Sum-Ping ST, England M. ICU sedation after coronary artery bypass graft surgery: dexmedetomidine-based versus propofol-based sedation regimens. J Cardiothorac Vasc Anesth 2003; 17: 576-84.

[34] Leino K, Hynynen M, Jalonen J, Salmenpera M, Scheinin H, Aantaa R et al. Renal effects of dexmedetomidine during coronary artery bypass surgery: a randomized placebo-controlled study. BMC Anesthesiol 2011; 11: 9. 